FSUJ-TPI-20/96

DIAS-STP-24/96

December 1996

\title{
Multi-scale Renormalization
}

\author{
C. Ford \\ Theor.-Phys. Institut, Universität Jena \\ Fröbelstieg 1 \\ D-07743 Jena \\ Germany \\ C. Wiesendangerf \\ Dublin Institute for Advanced Studies \\ 10 Burlington Road \\ Dublin 4 \\ Ireland
}

\begin{abstract}
The standard MS renormalization prescription is inadequate for dealing with multi-scale problems. To illustrate this we consider the computation of the effective potential in the Higgs-Yukawa model. It is argued that it is natural to employ a two-scale renormalization group. We give a modified version of a two-scale scheme introduced by Einhorn and Jones. In such schemes the beta functions necessarily contain potentially large logarithms of the RG scale ratios. For credible perturbation theory one must implement a large logarithms resummation on the beta functions themselves. We show how the integrability condition for the two RG equations allows one to perform this resummation.
\end{abstract}

\footnotetext{
${ }^{1}$ e-mail: cfo@tpi.uni-jena.de

${ }^{2}$ e-mail: wie@stp.dias.ie.
} 


\section{Introduction}

Consider the effective potential in the four-dimensional Higgs-Yukawa model defined by the Lagrangian

$$
\mathcal{L}=\frac{1}{2}\left(\partial_{\mu} \phi\right)^{2}-\frac{1}{2} m^{2} \phi^{2}-\frac{\lambda}{24} \phi^{4}+\bar{\psi} i \not \partial \psi+g \bar{\psi} \phi \psi+\Lambda
$$

where $\phi$ is a real scalar field and $\psi=\left(\psi_{1}, \ldots, \psi_{N}\right)^{T}$ are Dirac fields. Here $\Lambda$ is a "cosmological constant" term which enters non-trivially into the renormalization group equation for the effective potential [1]. It is well known how to perform a loopwise perturbative expansion of the effective potential [2], $V(\phi)=V^{\text {tree }}(\phi)+$ $\hbar V^{\text {-loop }}(\phi)+\hbar^{2} V^{2 \text {-loop }}(\phi)+\ldots$. Dimensional regularization together with (modified) minimal subtraction gives

$$
\begin{aligned}
V^{\text {tree }}(\phi) & =\frac{\lambda}{24} \phi^{4}+\frac{1}{2} m^{2} \phi^{2}-\Lambda \\
V^{1-\operatorname{loop}}(\phi) & =\frac{\left(m^{2}+\frac{1}{2} \lambda \phi^{2}\right)^{2}}{4(4 \pi)^{2}}\left[\log \frac{m^{2}+\frac{1}{2} \lambda \phi^{2}}{\mu^{2}}-\frac{3}{2}\right]-\frac{N g^{4} \phi^{4}}{(4 \pi)^{2}}\left[\log \frac{g^{2} \phi^{2}}{\mu^{2}}-\frac{3}{2}\right] .
\end{aligned}
$$

We have a $\log \frac{m^{2}+\frac{1}{2} \lambda \phi^{2}}{\mu^{2}}$ due to the "Higgs" loop and a $\log \frac{g^{2} \phi^{2}}{\mu^{2}}$ associated with the fermionic contribution to the one-loop potential. The two-loop potential $V^{2 \text {-loop }}(\phi)$ is quadratic in these logarithms, and in general the $n$-loop potential is a $n$th order polynomial in the two logarithms. Thus, for believable perturbation theory one must not only have "small" couplings $\hbar \lambda, \hbar g^{2}$, but the two logarithms must also be small. As was explained a long time ago by Coleman and Weinberg (CW) [3] one must make a ( $\phi$-dependent) choice of $\mu$ such that the logarithms are not too large. To relate the renormalized parameters at different scales one uses the renormalization group ( $\mathrm{RG}$ ). The CW procedure of RG "improving" the potential is equivalent to a resummation of the large logarithms in the perturbation series.

However, it is not too difficult to see that if $m^{2}+\frac{1}{2} \lambda \phi^{2} \gg g^{2} \phi^{2}$ (the heavy Higgs case) or $g^{2} \phi^{2} \gg m^{2}+\frac{1}{2} \lambda \phi^{2}$ (ie. heavy fermions) there is no choice of $\mu$ that will simultaneously render both logarithms small [4]. Thus, we are only able to implement the CW method when $m^{2}+\frac{1}{2} \lambda \phi^{2} \sim g^{2} \phi^{2}$, ie. when we have essentially a one-scale problem. In our opinion, the natural way to deal with this problem is to use a two-scale version of MS. A multi-scale version of MS was developed by Einhorn and Jones [5] (EJ). An alternative multi-scale approach based on the Callan-Symanzik equation has been outlined in refs. [6]. However, the EJ scheme had two drawbacks. Firstly, although one has several RG scales they do not "track" the relevant logarithms in an obvious way. Secondly, the beta functions contain logarithms of the RG scale ratios which render the perturbative beta functions useless when these ratios are large.

In this letter we present a simple modification of the EJ prescription where the RG scales naturally track the scales which appear logarithmically in the effective 
potential. As in the original EJ proposal one has potentially large logarithms in the beta functions. We argue that to deal with these logarithms one must implement a large logarithms resummation on the beta functions themselves. It is shown that the integrability condition for the two RG's allows one to perform this resummation.

\section{The Einhorn-Jones Approach}

Let us briefly recall the EJ multi-scale prescription. To motivate their idea consider the bare Lagrangian for our Higgs-Yukawa problem written in terms of the usual MS renormalized parameters.

$$
\begin{aligned}
\mathcal{L}_{\text {Bare }}= & \frac{1}{2} Z_{\phi}\left(\partial_{\mu} \phi\right)^{2}-\frac{1}{2} Z_{\phi} Z_{m^{2}} m^{2} \phi^{2}-\frac{1}{24} \mu^{\epsilon} Z_{\phi}^{2} Z_{\lambda} \lambda \phi^{4} \\
& +Z_{\psi} \bar{\psi} i \not \partial \psi+Z_{\psi} Z_{g^{2}}^{\frac{1}{2}} Z_{\phi}^{\frac{1}{2}} \mu^{\frac{1}{2} \epsilon} g \bar{\psi} \phi \psi+\Lambda+\mu^{-\epsilon}\left(Z_{\Lambda}-1\right) m^{4} \lambda^{-1}
\end{aligned}
$$

where $\epsilon=4-d$ is the dimensional continuation parameter, and all the $Z$. factors have the form $Z$. $=1+$ pole terms only. Notice that the MS RG scale $\mu$ enters eqn. (3) in three places. The EJ idea was simply to replace the three occurrences of $\mu$ in

(3) with three independent RG scales $\kappa_{1}, \kappa_{2}, \kappa_{3}$, so that

$$
\begin{aligned}
\lambda_{B} & =\kappa_{1}^{\epsilon} Z_{\lambda} \lambda, \quad g_{B}^{2}=\kappa_{2}^{\epsilon} Z_{g^{2}} g^{2}, \quad \Lambda_{B}=\Lambda+\kappa_{3}^{-\epsilon}\left(Z_{\Lambda}-1\right) m^{4} \lambda^{-1} \\
m_{B}^{2} & =Z_{m^{2}} m^{2}, \quad \phi_{B}=Z_{\phi}^{\frac{1}{2}} \phi, \quad \psi_{B}=Z_{\psi}^{\frac{1}{2}} \psi .
\end{aligned}
$$

As in standard MS the $Z$. factors are defined by the requirement that the effective action is finite when written in terms of the renormalized parameters and the restriction that the $Z$. factors have the form $Z$. $=1+$ pole terms only. Note that the $Z$. factors will not be the same as the MS $Z$. factors (except where $\kappa_{1}=\kappa_{2}=\kappa_{3}$ ). In the EJ scheme the $Z$.'s contain logarithms of the RG scale ratios.

We now have three separate RG equations associated with the independent variations of the three RG scales. We also have three sets of beta functions

$$
{ }_{i} \beta_{\lambda}=\kappa_{i} \frac{d}{d \kappa_{i}} \lambda ; \quad i=1,2,3
$$

and similarly for the other parameters. It is straightforward to compute the oneloop RG functions in the EJ scheme. For example, the one-loop beta functions for the two couplings are

$$
\begin{aligned}
& { }_{2} \beta_{g^{2}}^{1 \text {-loop }}=\frac{(4 N+6) \hbar g^{4}}{(4 \pi)^{2}},{ }_{1} \beta_{g^{2}}^{1-\text { loop }}={ }_{3} \beta_{g^{2}}^{1 \text {-loop }}=0, \\
& { }_{1} \beta_{\lambda}^{1 \text {-loop }}=\frac{\hbar}{(4 \pi)^{2}}\left(3 \lambda^{2}+48 N g^{4}\right),{ }_{2} \beta_{\lambda}^{1 \text {-loop }}=\frac{\hbar}{(4 \pi)^{2}}\left(8 N \lambda g^{2}-96 N g^{4}\right),{ }_{3} \beta_{\lambda}^{1-\text { loop }}=0 .(6)
\end{aligned}
$$

However, if one were to compute the two-loop beta functions, one would find terms proportional to $\log \frac{\kappa_{1}}{\kappa_{2}}$, and in general the $n$-loop RG functions contain $\log ^{n-1} \frac{\kappa_{1}}{\kappa_{2}}$ 
terms (as well as lower powers of the logarithm). Therefore, unlike in standard MS we cannot trust the perturbative $R G$ functions.

In the EJ scheme the one-loop potential is

$$
\begin{aligned}
V^{\text {1-loop }}= & \frac{\left(m^{2}+\frac{1}{2} \lambda \phi^{2}\right)^{2}}{4(4 \pi)^{2}}\left(\log \frac{m^{2}+\frac{1}{2} \lambda \phi^{2}}{\kappa_{1}^{2}}-\frac{3}{2}\right) \\
& -\frac{N g^{4} \phi^{4}}{(4 \pi)^{2}}\left(\log \frac{g^{2} \phi^{2}}{\kappa_{2}^{2}}+\log \frac{\kappa_{1}^{2}}{\kappa_{2}^{2}}-\frac{3}{2}\right)+\frac{m^{4}}{2(4 \pi)^{2}} \log \frac{\kappa_{1}}{\kappa_{3}}
\end{aligned}
$$

whereas what we want is a two-scale scheme where the one-loop potential is

$$
V^{1 \text {-loop }}=\frac{\left(m^{2}+\frac{1}{2} \lambda \phi^{2}\right)^{2}}{4(4 \pi)^{2}}\left(\log \frac{m^{2}+\frac{1}{2} \lambda \phi^{2}}{\kappa_{1}^{2}}-\frac{3}{2}\right)-\frac{N g^{4} \phi^{4}}{(4 \pi)^{2}}\left(\log \frac{g^{2} \phi^{2}}{\kappa_{2}^{2}}-\frac{3}{2}\right) .
$$

Attaching $R G$ scales to the kinetic terms

The reason for this mismatch is that in the EJ scheme the RG scales are attached to the coupling constant terms in the Lagrangian, but the arguments of the logarithms in the effective potential are associated with the kinetic part of the action. However, there is no reason why independent RG scales should not be "attached" to the kinetic terms. That is, instead of (3) we propose to start with

$$
\begin{aligned}
\mathcal{L}_{\text {Bare }}= & \frac{1}{2} \kappa_{1}{ }^{-\frac{1}{2} \epsilon} Z_{\phi}\left(\partial_{\mu} \phi\right)^{2}-\frac{1}{2} Z_{\phi} Z_{m^{2}} m^{2} \phi^{2}-\frac{Z_{\lambda} Z_{\phi}^{2}}{24} \lambda \phi^{4} \\
& +\kappa_{2}{ }^{-\frac{1}{4} \epsilon} Z_{\psi} \bar{\psi} i \not \partial \psi+Z_{g^{2}}^{\frac{1}{2}} Z_{\psi} Z_{\phi}^{\frac{1}{2}} g \bar{\psi} \phi \psi+\Lambda+\left(Z_{\Lambda}-1\right) m^{4} \lambda^{-1} .
\end{aligned}
$$

As before we insist that the renormalized couplings $\lambda$ and $g$ are dimensionless. However, by altering the dimensions of the renormalized dimensionful objects $\mathrm{m}^{2}$, $\phi$ and $\psi$ we can reshuffle the RG scales into the kinetic terms only. Again we require that the $Z$. factors are of the form $Z$. $=1+$ pole terms only.

From (9) one can read off the relation between the bare and renormalized parameters

$$
\begin{array}{ll}
\lambda_{B}=\kappa_{1}{ }^{\epsilon} Z_{\lambda} \lambda, \quad g_{B}^{2}=\kappa_{1}{ }^{\frac{1}{2} \epsilon} \kappa_{2}^{\frac{1}{2} \epsilon} Z_{g^{2}} g^{2}, & m_{B}^{2}=\kappa_{1}^{\frac{1}{2} \epsilon} Z_{m^{2}} m^{2} \\
\phi_{B}=\kappa_{1}{ }^{-\frac{1}{4} \epsilon} Z_{\phi}^{\frac{1}{2}} \phi, \quad \psi_{B}=\kappa_{2}^{-\frac{1}{8} \epsilon} Z_{\psi}^{\frac{1}{2}} \psi, & \Lambda_{B}=\Lambda+\left(Z_{\Lambda}-1\right) m^{4} \lambda^{-1} .
\end{array}
$$

In this "modified" EJ approach the one-loop effective potential is indeed given by (8). It is straightforward to obtain the beta functions in terms of the $Z$. factors. As in standard MS one only needs the simple pole terms in the $Z$.'s

$$
\begin{aligned}
{ }_{1} \beta_{\lambda} & =-\epsilon \lambda+\lambda\left(\lambda \partial_{\lambda}+\frac{1}{2} g^{2} \partial_{g^{2}}\right) z_{\lambda} & { }_{1} \beta_{g^{2}} & =-\frac{1}{2} \epsilon g^{2}+g^{2}\left(\lambda \partial_{\lambda}+\frac{1}{2} g^{2} \partial_{g^{2}}\right) z_{g^{2}} \\
{ }_{2} \beta_{\lambda} & =\frac{1}{2} \lambda g^{2} \partial_{g^{2}} z_{\lambda} & { }_{2} \beta_{g^{2}} & =-\frac{1}{2} \epsilon g^{2}+\frac{1}{2} g^{4} \partial_{g^{2}} z_{g^{2}}, \\
{ }_{1} \beta_{m^{2}} & =-\frac{1}{2} \epsilon m^{2}+m^{2}\left(\lambda \partial_{\lambda}+\frac{1}{2} g^{2} \partial_{g^{2}}\right) z_{m^{2}} & { }_{1} \beta_{\Lambda} & =\lambda^{-1} m^{4}\left(\lambda \partial_{\lambda}+\frac{1}{2} g^{2} \partial_{g^{2}}\right) z_{\Lambda} \\
{ }_{2} \beta_{m^{2}} & =\frac{1}{2} m^{2} g^{2} \partial_{g^{2}} z_{m^{2}} & { }_{2} \beta_{\Lambda} & =\frac{1}{2} \lambda^{-1} m^{4} g^{2} \partial_{g^{2}} z_{\Lambda} \\
{ }_{1} \beta_{\phi} & =\frac{1}{4} \epsilon+\frac{1}{2}\left(\lambda \partial_{\lambda}+\frac{1}{2} g^{2} \partial_{g^{2}}\right) z_{\phi} & { }_{1} \beta_{\psi} & =\frac{1}{2}\left(\lambda \partial_{\lambda}+\frac{1}{2} g^{2} \partial_{g^{2}}\right) z_{\psi} \\
{ }_{2} \beta_{\phi} & =\frac{1}{4} g^{2} \partial_{g^{2}} z_{\phi} & { }_{2} \beta_{\psi} & =\frac{1}{8} \epsilon+\frac{1}{4} g^{2} \partial_{g^{2}} z_{\psi} .
\end{aligned}
$$


Here $z$. denotes the coefficient of $1 / \epsilon$ in $Z$. To compute the one-loop beta functions one needs the one-loop contribution to the $z$.'s which are identical to the MS $z$.'s. However, at higher loops the $z$.'s (and hence the beta-functions) contain logarithms of the $\mathrm{RG}$ scale ratios. When these logarithms are large the one-loop multi-scale RG functions cannot be trusted. To get reliable approximations to the beta functions we must implement a large logarithms resummation on the beta functions themselves.

\section{Integrability of the two $R G$ equations}

The effective potential satisfies two RG equations associated with the independent variations of the two $R G$ scales

$$
\mathcal{D}_{i} V=0 ; \quad i=1,2
$$

where

$$
\mathcal{D}_{i}=\kappa_{i} \frac{\partial}{\partial \kappa_{i}}+{ }_{i} \beta_{g^{2}} \frac{\partial}{\partial g^{2}}+{ }_{i} \beta_{\lambda} \frac{\partial}{\partial \lambda}+{ }_{i} \beta_{m^{2}} \frac{\partial}{\partial m^{2}}+{ }_{i} \beta_{\Lambda} \frac{\partial}{\partial \Lambda}+{ }_{i} \beta_{\phi} \phi \frac{\partial}{\partial \phi}
$$

The integrability condition for these two equations is simply

$$
\left[\mathcal{D}_{1}, \mathcal{D}_{2}\right]=0
$$

ie. independent variations of the two RG scales commute. Recently, it has been argued that in any multi-scale scheme the integrability condition imposes a strong constraint on the form of the multi-scale RG functions [7]. Here we show that for EJ type schemes this constraint is strong enough to implement a large logs resummation on the beta functions.

If we now insert the expressions for the multi-scale beta functions in terms of the $z$.'s into the integrability condition (14) we obtain a set of (non-linear) partial differential equations for the $z$.'s.

$$
\begin{aligned}
0= & (4 \pi)^{-2} \hbar \partial_{t}\left(g^{2} \partial_{g^{2}}+\lambda \partial_{\lambda}\right) z_{\lambda}+\frac{1}{2} \lambda^{2} g^{2}\left(\partial_{\lambda} z_{\lambda}\right) \partial_{g^{2}} \partial_{\lambda} z_{\lambda}-\frac{1}{2} \lambda g^{2}\left(\partial_{g^{2}} z_{\lambda}\right) \partial_{\lambda} z_{\lambda} \\
& -\frac{1}{2} g^{2} \lambda^{2}\left(\partial_{g^{2}} z_{\lambda}\right) \partial_{\lambda}{ }^{2} z_{\lambda}+\frac{1}{2} g^{2} \lambda\left(\partial_{\lambda} z_{g^{2}}\right) \partial_{g^{2}} z_{\lambda}+\frac{1}{2} g^{4} \lambda\left(\partial_{\lambda} z_{g^{2}}\right) \partial_{g^{2}}{ }^{2} z_{\lambda} \\
& -\frac{1}{2} g^{4} \lambda\left(\partial_{g^{2}} z_{g^{2}}\right) \partial_{\lambda} \partial_{g^{2}} z_{\lambda}, \\
0= & (4 \pi)^{-2} \hbar \partial_{t}\left(g^{2} \partial_{g^{2}}+\lambda \partial_{\lambda}\right) z_{g^{2}}+\frac{1}{2} \lambda g^{2}\left(\partial_{\lambda} z_{g^{2}}\right) \partial_{g^{2}} z_{g^{2}}+\frac{1}{2} \lambda g^{4}\left(\partial_{\lambda} z_{g^{2}}\right) \partial_{g^{2}}^{2} z_{g^{2}} \\
& -\frac{1}{2} \lambda g^{4}\left(\partial_{g^{2}} z_{g^{2}}\right) \partial_{\lambda} \partial_{g^{2}} z_{g^{2}}+\frac{1}{2} \lambda^{2} g^{2}\left(\partial_{\lambda} z_{\lambda}\right) \partial_{\lambda} \partial_{g^{2}} z_{g^{2}}-\frac{1}{2} \lambda g^{2}\left(\partial_{g^{2}} z_{\lambda}\right) \partial_{\lambda} z_{g^{2}} \\
& -\frac{1}{2} \lambda^{2} g^{2}\left(\partial_{g^{2}} z_{\lambda}\right) \partial_{\lambda}^{2} z_{g^{2}}, \\
0 & (4 \pi)^{-2} \hbar \partial_{t}\left(g^{2} \partial_{g^{2}}+\lambda \partial_{\lambda}\right) z_{m^{2}}+\frac{1}{2} \lambda^{2} g^{2}\left(\partial_{\lambda} z_{\lambda}\right) \partial_{g^{2}} z_{m^{2}}-\frac{1}{2} g^{2}\left(\partial_{g^{2}} z_{\lambda}\right) \partial_{\lambda} z_{m^{2}} \\
& -\frac{1}{2} \lambda g^{2}\left(\partial_{\lambda} z_{\lambda}\right) \partial_{\lambda}^{2} z_{m^{2}}+\frac{1}{2} \lambda g^{2}\left(\partial_{\lambda} z_{g^{2}}\right) \partial_{g^{2}} z_{m^{2}}+\frac{1}{2} \lambda g^{4}\left(\partial_{\lambda} z_{g^{2}}\right) \partial_{g^{2}}^{2} z_{m^{2}} \\
& -\frac{1}{2} \lambda g^{4}\left(\partial_{g^{2}} z_{g^{2}}\right) \partial_{\lambda} \partial_{g^{2}} z_{m^{2}},
\end{aligned}
$$

where $(4 \pi)^{2} t=\hbar \log \left(\kappa_{1} / \kappa_{2}\right)$. One can obtain similar equations for $z_{\phi}, z_{\Lambda}$ and $z_{\psi}$. These equations which are exact to all orders in perturbation theory, together with 
the boundary conditions that $z$. collapses to the MS result at $\kappa_{1}=\kappa_{2}$, fully determine all the logarithmic terms in the $z$.'s (and hence the beta functions). To sum the leading logarithms (LL) in the $z$.'s we must solve equations (15) using the one-loop MS $z$.'s as boundary conditions at $t=0$ (ie. $\left.\kappa_{1}=\kappa_{2}\right)$

$$
\begin{array}{lll}
(4 \pi)^{2} z_{\lambda}^{1 \text {-loop }}=\hbar\left(3 \lambda+8 N g^{2}-48 N g^{4} \lambda^{-1}\right), & (4 \pi)^{2} z_{g^{2}}^{1 \text {-loop }}=(4 N+6) \hbar g^{2}, \\
(4 \pi)^{2} z_{m^{2}}^{1 \text {-lop }}=\hbar m^{2}\left(\lambda+4 N g^{2}\right), & (4 \pi)^{2} z_{\Lambda}^{1 \text {-loop }}=\frac{1}{2} \hbar \lambda m^{4}, \\
(4 \pi)^{2} z_{\phi}^{1-\text { loop }}=-2 N \hbar g^{2}, & (4 \pi)^{2} z_{\psi}^{1 \text {-loop }}=\hbar g^{2}
\end{array}
$$

In general we do not know how to solve equations of the type (15). However, for the LL calculation the following ansatz is applicable

$$
(4 \pi)^{2} z_{\lambda}^{\mathrm{LL}}=\hbar\left[\lambda a(s)+g^{2} b(s)+g^{4} \lambda^{-1} c(s)\right]
$$

where $s=g^{2} t=\hbar g^{2}(4 \pi)^{-2} \log \left(\kappa_{1} / \kappa_{2}\right)$, and $z_{g^{2}}^{\mathrm{LL}}, z_{\phi}^{\mathrm{LL}}, z_{\psi}^{\mathrm{LL}}$ are identical to the one-loop results (16) (ie. the RG functions for $g^{2}, \phi$ and $\psi$ contain no leading logarithms). The functions $a(s), b(s), c(s)$ satisfy the following (coupled) ordinary differential equations

$$
\begin{aligned}
s a^{\prime \prime}(s)+2 a^{\prime}(s) & =\frac{1}{2} a(s)\left(b(s)+s b^{\prime}(s)\right)+(2 N+3) s a^{\prime}(s), \\
s b^{\prime \prime}(s)+2 b^{\prime}(s) & =s a^{\prime}(s) c(s)+\left(2 c(s)+s c^{\prime}(s)\right) a(s), \\
s c^{\prime \prime}(s)+2 c^{\prime}(s) & =\frac{1}{2} c(s)\left(b(s)+s b^{\prime}(s)\right)-(2 N+3)\left(2 c(s)+s c^{\prime}(s)\right),
\end{aligned}
$$

where $^{\prime}=d / d s$, and the initial values are $a(0)=3, b(0)=8 N, c(0)=-48 N$. The corresponding RG functions are

$$
\begin{gathered}
(4 \pi)^{2}{ }_{1} \beta_{g^{2}}^{\mathrm{LL}}=(2 N+3) \hbar g^{4}, \quad(4 \pi)^{2}{ }_{1} \beta_{\phi}^{\mathrm{LL}}=-N \hbar g^{2}, \quad(4 \pi)^{2}{ }_{1} \beta_{\psi}^{\mathrm{LL}}=\frac{1}{2} \hbar g^{2}, \\
(4 \pi)_{2}^{2} \beta_{g^{2}}^{\mathrm{LL}}=(2 N+3) \hbar g^{4}, \quad(4 \pi)^{2}{ }_{2} \beta_{\phi}^{\mathrm{LL}}=-N \hbar g^{2}, \quad(4 \pi)^{2}{ }_{2} \beta_{\psi}^{\mathrm{LL}}=\frac{1}{2} \hbar g^{2}, \\
(4 \pi)^{2}{ }_{1} \beta_{\lambda}^{\mathrm{LL}}=\hbar \lambda^{2}\left(a(s)+\frac{1}{2} s a^{\prime}(s)\right)+\frac{1}{2} \hbar \lambda g^{2}\left(b(s)+s b^{\prime}(s)\right)+\frac{1}{2} \hbar g^{4} s c^{\prime}(s), \\
(4 \pi)^{2}{ }_{2} \beta_{\lambda}^{\mathrm{LL}}=\frac{1}{2} \hbar \lambda^{2} s a^{\prime}(s)+\frac{1}{2} \hbar \lambda g^{2}\left(b(s)+s b^{\prime}(s)\right)+\frac{1}{2} \hbar g^{4}\left(2 c(s)+s c^{\prime}(s)\right) .
\end{gathered}
$$

We have seen that $z_{g^{2}}^{\mathrm{LL}}, z_{\phi}^{\mathrm{LL}}$, and $z_{\psi}^{\mathrm{LL}}$ are trivial, while $z_{\lambda}^{\mathrm{LL}}$ has the form (17). However, the problem of computing $z_{m^{2}}^{\mathrm{LL}}$ and $z_{\Lambda}^{\mathrm{LL}}$ is more tricky. They satisfy the following partial differential equations

$$
\begin{aligned}
0= & g^{2}\left(s \partial_{s}^{2}+2 \partial_{s}\right) z_{m^{2}}^{\mathrm{LL}}+\frac{1}{2} g^{2}\left(\lambda^{2} a(s)-g^{4} c(s)\right) \partial_{\lambda} \partial_{g^{2}} z_{m^{2}}^{\mathrm{LL}}-(2 N+3) \lambda g^{4} \partial_{\lambda} \partial_{g^{2}} z_{m^{2}}^{\mathrm{LL}} \\
& -\left[\lambda^{2} a^{\prime}(s) s+\lambda g^{2}\left(b(s)+b^{\prime}(s) s\right)+g^{4}\left(2 c(s)+c^{\prime}(s) s\right)\right]\left(\partial_{\lambda}+\lambda \partial_{\lambda}^{2}\right) z_{m^{2}}^{\mathrm{LL}} \\
0= & \lambda^{-1} g^{2}\left(s \partial_{s}^{2}+2 \partial_{s}\right) z_{\Lambda}^{\mathrm{LL}}+\left(\lambda^{2} a(s)-g^{4} c(s)\right)\left[\frac{1}{2} \lambda^{-1} g^{2} \partial_{\lambda} \partial_{g^{2}} z_{\Lambda}^{\mathrm{LL}}-\frac{1}{2} \lambda^{-2} g^{2} \partial_{g^{2}} z_{\Lambda}^{\mathrm{LL}}\right] \\
& -\left[\lambda^{2} a^{\prime}(s) s+\lambda g^{2}\left(b(s)+b^{\prime}(s) s\right)+g^{4}\left(2 c(s)+c^{\prime}(s) s\right)\right] \partial_{\lambda}^{2} z_{\Lambda}^{\mathrm{LL}} \\
& -(2 N+3) \lambda g^{4} \partial_{\lambda} \partial_{g^{2}} z_{\Lambda}^{\mathrm{LL}}+g^{2}\left(\partial_{\lambda} z_{m^{2}}^{\mathrm{LL}}\right) \partial_{g^{2}} z_{\Lambda}^{\mathrm{LL}}-g^{2}\left(\partial_{g^{2}} z_{m^{2}}^{\mathrm{LL}}\right) \partial_{\lambda} z_{\Lambda}^{\mathrm{LL}} .
\end{aligned}
$$


Although we do not have closed forms for $z_{\lambda}^{\mathrm{LL}}, z_{m^{2}}^{\mathrm{LL}}$ and $z_{\Lambda}^{\mathrm{LL}}$ in the LL approximation, it is easy to check that for the special cases $N=0$ and $N \rightarrow \infty$ there are no logarithms in the $z$.'s. This is what we would expect since here we are dealing with single scale problems.

\section{Discussion}

We have presented a modification of the EJ multiscale prescription where the RG scales naturally track the logarithms present in the perturbation series for the effective potential. This involves the attachment of independent RG scales to the kinetic terms in the action rather than to the interaction terms. We have illustrated our proposal using a simple two-scale Higgs-Yukawa model. It is straightforward to apply this approach to other renormalizable theories ?

As in the EJ prescription there are potentially large logarithms in the beta functions. We have shown that the integrability condition for the two RG equations is sufficient (in principle) to implement a large logarithms resummation on the beta functions. However we have seen that even in the simple Higgs-Yukawa model, the calculation of the LL beta functions is quite involved (one needs to solve the system of ODE's (18) to determine the ${ }_{i} \beta_{\lambda}^{\mathrm{LL}}$, while ${ }_{i} \beta_{m^{2}}^{\mathrm{LL}}$ and ${ }_{i} \beta_{\Lambda}^{\mathrm{LL}}$ require a solution of eqns. (20)). An analytic or numerical solution of these equations would be helpful.

\section{Acknowledgements}

We are grateful to D. O' Connor, J.M. Pawlowski and C.R. Stephens for stimulating discussions. C. F. thanks DIAS for warm hospitality and financial support. C. W. has been partially supported by Schweizerischer Nationalfonds.

\section{References}

[1] B. Kastening, Phy. Lett. B 283, 287 (1992);

M. Bando, T. Kugo, N. Maekawa and H. Nakano, Phys. Lett. B 301, 83 (1992); I.L. Buchbinder, S.D. Odintsov and I.L. Shapiro, Effective Action in Quantum Gravity (Institute of Physics Publishing, Bristol, 1992).

[2] R. Jackiw, Phys. Rev. D 10, 1686 (1974).

[3] S. Coleman and E. Weinberg, Phys. Rev. D 9, 1888 (1973).

[4] M. Bando, T. Kugo, N. Maekawa and H. Nakano, Prog. Theor. Phys. 90, 485 (1993).

\footnotetext{
${ }^{3}$ The situation is a little more complicated in gauge theories where one has interactions involving derivatives. Moreover, one should ensure that the attachment of independent RG scales does not spoil gauge invariance.
} 
[5] M.B. Einhorn and D.R.T. Jones, Nucl. Phys. B 230 [FS10], 261 (1984).

[6] K. Nishijima, Prog. Theor. Phys. 88, 993 (1992); Prog. Theor. Phys. 89, 917 (1993).

[7] C. Ford and C. Wiesendanger, Phys. Rev. D in press, hep-ph/9604392;

C. R. Stephens, hep-th/9611062. 